\title{
Major Changes of von Willebrand Factor Multimer Distribution in Cirrhotic Patients with Stable Disease or Acute Decompensation
}

\author{
Eszter Palyu ${ }^{1, *}$ Jolan Harsfalvi ${ }^{2, *}$ Tamas Tornai ${ }^{1}$ Maria Papp ${ }^{1} \quad$ Miklos Udvardy ${ }^{3}$ \\ Katalin Szekeres-Csiki ${ }^{3}$ Lajos Pataki ${ }^{3}$ Karen Vanhoorelbeke ${ }^{4}$ Hendrik B. Feys ${ }^{5,6}$ Hans Deckmyn ${ }^{4}$ \\ Istvan Tornai ${ }^{1}$
}

1 Division of Gastroenterology, Department of Internal Medicine, Faculty of Medicine, University of Debrecen, Debrecen, Hungary

${ }^{2}$ Department of Biophysics and Radiation Biology, Semmelweis University, Budapest, Hungary

${ }^{3}$ Department of Laboratory Medicine, Faculty of Medicine, University of Debrecen, Debrecen, Hungary

${ }^{4}$ Laboratory for Thrombosis Research, IRF Life Science, KU Leuven Kulak, Kortrijk, Belgium

${ }^{5}$ Transfusion Research Center, Belgian Red Cross-Flanders, Ghent, Belgium

6 Faculty of Medicine and Health Sciences, Ghent University, Ghent, Belgium

\begin{abstract}
Address for correspondence Istvan Tornai, MD, PhD, Division of Gastroenterology, Department of Internal Medicine, Faculty of Medicine, University of Debrecen, Nagyerdei krt. 98, H-4032 Debrecen, Hungary (e-mail: itornai@med.unideb.hu).
\end{abstract}

Thromb Haemost 2018;118:1397-1408.

\author{
Abstract \\ Keywords \\ - cirrhosis \\ - acute \\ decompensation \\ - systemic \\ inflammation \\ - von Willebrand factor \\ multimers \\ - thrombotic micro- \\ angiopathy
}

Background There is an unstable balance between pro- and anti-haemostatic processes in patients with cirrhosis. We hypothesized, that in patients with acute decompensation (AD) the major alterations of von Willebrand factor (VWF) could contribute to the prothrombotic situation as compared to patients with stable (ST) cirrhosis.

Patients and Methods We analysed different parameters of VWF, including detailed multimer distribution by densitometry and platelet adhesion, together with a disintegrin-like and metalloproteinase with thrombospondin type-1 motifs 13 (ADAMTS13) activity and antigen and C-reactive protein (CRP) levels in patients with ST cirrhosis $(n=99)$, with $\operatorname{AD}(n=54)$ and controls $(n=92)$.

Results VWF antigen, ristocetin co-factor as well as collagen-binding activities were elevated in both cirrhotic groups in a stepwise manner. There was a decrease in high and an increase in low molecular weight multimer ratios in the majority of ST cirrhosis. However, in 24 out of 54 AD patients, ultra-large VWF multimers (ultra-large molecular weight multimers [ULMWM]) were found. ADAMTS13 activity in ST and AD patients without ULMWM was similar to controls (median [interquartile range; IQR]\%: 98 [67-132] and 91 [60-110] vs. 106 [88-117], respectively). The presence of ULMWM in AD patients was associated with low ADAMTS13 activity [33 (24-49)\%] and high CRP level [23 (7.1-83.6) mg/L]. Adhesion of normal platelets showed a stepwise increase in the presence of cirrhotic plasmas, reaching the highest level in $A D$ patients with ULMWM.

Conclusion Characteristic changes of VWF parameters are seen in ST cirrhosis. In AD patients, highly increased VWF and reduced ADAMTS13 activity could be found, along with the presence of ULMWM, which are possible markers and contributors of the disease progression.

* These authors contributed equally to this work and should be considered as first authors.

received

March 21, 2018

accepted after revision May 17, 2018
DOI https://doi.org/

10.1055/s-0038-1661393. ISSN 0340-6245. (c) 2018 Georg Thieme Verlag KG Stuttgart · New York
License terms

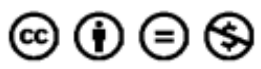




\section{Introduction}

von Willebrand factor (VWF) is a large, multimeric protein, playing a central role in platelet adhesion and primary haemostasis. The level of VWF is increased in patients with cirrhosis and correlates with the severity of the disease. There is a tightly regulated balance between pro-haemostatic and anti-haemostatic processes, and this might actually also be maintained in patients with cirrhosis. ${ }^{1,2}$ Platelet abnormalities can be compensated by elevated VWF levels as was suggested previously. ${ }^{3,4}$ There are studies where also a close correlation of VWF level and portal hypertension as well as mortality has been described. ${ }^{5,6}$

The activity of VWF is strictly regulated by the VWFcleaving protease a disintegrin-like and metalloproteinase with thrombospondin type-1 motifs $\underline{13}$ (ADAMTS13), as the reactivity of VWF towards platelets is proportional to its multimeric size. ${ }^{7}$ In patients with either congenital or acquired deficiency of ADAMTS13, ultra-large VWF multimers are present leading to thrombotic micro-angiopathy, for example, thrombotic thrombocytopenic purpura (TTP). ${ }^{8}$ ADAMTS13 is synthesized by hepatic stellate cells ${ }^{9,10}$ that are generally activated in liver cirrhosis. ${ }^{11}$ Previously variable activity and antigen levels of ADAMTS13 have been reported in patients with liver cirrhosis. Mannucci et $\mathrm{al}^{12}$ and Feys et $\mathrm{al}^{13}$ discovered that ADAMTS13 activity is significantly reduced in advanced liver disease.

The previously demonstrated pro-thrombotic tendency, endothelial dysfunction together with systemic inflammation and bacterial infections can play a major role in the worsening of cirrhosis. ${ }^{14-18}$ The development of bacterial infections like spontaneous bacterial peritonitis, hepatic encephalopathy (HE), gastrointestinal (GI) bleeding and/or rapid accumulation of ascites in patients with previously stable disease are collectively referred to as acute decompensation (AD) as was defined in the CANONIC study. ${ }^{19}$

There are no solid data, however, about the characteristics of VWF parameters, like antigen level, functional activity, multimeric structure as well as ADAMTS13 in cirrhotic patients with AD as compared to stable (ST) cirrhosis. Since VWF is an acute phase protein, the alterations of this multimeric protein as well as ADAMTS13 seem to be important especially in an already rebalanced situation.

In this study, we aimed to analyse the quantitative parameters of VWF, including VWF antigen (VWF:Ag) and propeptide (VWFpp) levels, VWF ristocetin co-factor activity (VWF:RCo), collagen-binding activity (VWF:CB) as well as ADAMTS13 antigen and activity levels in controls and in patients with cirrhosis either in a stable outpatient cohort or hospitalized due to AD. Furthermore, we performed a quantitative multimeric analysis of VWF as well as platelet adhesion assay in the plasma of these patients. Since bacterial infections that elicit acute phase reactions are frequent in our cirrhotic patients, ${ }^{15,20-22}$ we also considered the association of systemic inflammation and VWF parameters. We wanted to clarify the effect of $A D$ and systemic inflammation in patients with cirrhosis on VWF parameters as well as on ADAMTS13 activity. Furthermore, we wanted to see whether the presence of a pro- thrombotic state can be confirmed in these patients, which also can contribute to the disease progression.

\section{Patients and Methods}

\section{Patients}

One hundred fifty-three well-characterized patients with cirrhosis of various causes were included consecutively from our in- and outpatient gastroenterology department into this study. The female/male ratio was $75 / 78$, and the median age was 56 (range: $24-80$ ) years. The diagnosis was based on clinical, biochemical and imaging investigations as well as on liver biopsy when available. The aetiology of cirrhosis was alcoholic liver disease $(n=106)$, chronic viral hepatitis C or B $(n=34)$ and others, like primary biliary cholangitis (previously cirrhosis), autoimmune hepatitis or cryptogenic cirrhosis $(n=13)$. This cohort comprises 99 outpatients with ST cirrhosis and 54 hospitalized subjects due to an $\mathrm{AD}$ episode. There was no patient included into both groups due to disease progression. The presence of acute-on-chronic liver failure (ACLF) was evaluated retrospectively within the $\mathrm{AD}$ group (i.e. additional presence of organ failure(s)), after its definition was published in the CANONIC study. ${ }^{19}$ Patients with hepatocellular carcinoma were excluded, as it can influence the levels of VWF:Ag and ADAMTS13..$^{23,24}$ None of the patients enrolled to this study received fresh frozen plasma, blood transfusion or platelet concentrate, nor did they use aspirin or any non-steroidal anti-inflammatory drugs for 2 weeks prior to the time of inclusion. Ninetytwo healthy age-matched volunteers served as controls.

\section{Methods}

Blood samples obtained by venipuncture from the antecubital vein into $3.2 \%$ sodium citrate $(9: 1, \mathrm{v} / \mathrm{v})$ were centrifuged twice $(2,000 \times g$ for 15 minutes), snap frozen in aliquots and stored at $-70^{\circ} \mathrm{C}$ until use.

C-reactive protein (CRP) was determined using the Integra 700 automated analyzer system (Roche, Basel, Switzerland), where $10 \mathrm{mg} / \mathrm{L}$ cut-off value-proved to be the most accurate for prediction of infection-free survival, ${ }^{15}$ and therefore we used this level in this trial.

VWF:Ag was determined using polyclonal antibody against VWF for coating and its horseradish peroxidase-labelled product for detection in an enzyme-linked immunosorbent assay (ELISA). ${ }^{25}$ Both antibodies were purchased from Dako (Glostrup, Denmark). VWF:CB was determined as was described in our previous publication. ${ }^{26}$ In short, human type III collagen (Sigma-Aldrich, St. Louis, Missouri, United States), $20 \mu \mathrm{g} / \mathrm{mL}$ in sodium phosphate-buffered saline (PBS), pH 7.4 was used for coating, followed by washing with PBS containing 0.1\% Tween-20 (PBS-T). Each well was then incubated with $100 \mu \mathrm{L}$ of plasma diluted in PBS-T at room temperature. VWF:Ag and VWF:CB measurements were performed in parallel using the same plasma dilutions. The amount of VWF bound to collagen was detected using the same horseradish peroxidase-labelled polyclonal antibody against VWF (Dako). The World Health Organisation (WHO) 2nd International Standard von Willebrand Factor, Concentrate, National Institute for 
Biological Standards and Control (NIBSC) code: 09/182 was used for calibration of these methods (NIBSC, UK). VWF:RCo was determined by using Ristocetin reagent that contained lyophilized platelets (Helena BioSciences, Sunderland, UK) in a Chrono-Log 810-CA lumi-aggregometer (Chronolog, Havertown, Pennsylvania, United States). VWFpp level was measured by ELISA kit using mouse antibody pair clones CLB-Pro 35 and CLB-Pro 14.3 (Cell Sciences, Canton, Massachusetts, United States), according to the manufacturer's instruction.

ADAMTS13 activity was determined by fluorescein resonance energy transfer analysis, ${ }^{27}$ using the TECHNOZYME ADAMTS-13 kit (Technoclone, Vienna, Austria), according to the instruction of the manufacturer. ADAMTS13 antigen levels were measured by ELISA as previously published. ${ }^{13}$ ADAMTS13 antigen level in pooled normal plasma was set at $100 \%$.

Multimeric analysis of VWF was determined as previously described. ${ }^{28}$ Briefly, VWF multimers were separated by sodium dodecyl sulphate (SDS) 0.8\% agarose gel electrophoresis, followed by Western blotting. Bands were visualized by horseradish peroxidase-labelled polyclonal antibody against VWF (Dako) and 3,3'-diaminobenzidine substrate (Sigma-Aldrich). The first 5 bands were considered as low, bands 6 to 10 as intermediate and larger than 10, including the area where VWF did not resolve into bands, as high molecular weight multimers (LMWM, IMWM, HMWM), respectively, as was previously described. ${ }^{29}$ Digital images of the bands were obtained by a GS-800 calibrated densitometer and processed by its QuantityOne software (Bio-Rad Laboratories, Richmond, California, United States). The proportions of the three groups of bands were calculated from the area under the curve (AUC), constructed for all bands. Additionally, all samples were separated on $0.65 \%$ gels too and visually analysed for the presence of ultralarge molecular weight multimers (ULMWM) within the HMWM. ULMWM were defined as the high molecular weight bands not seen in controls. ${ }^{29}$ The quantity of VWF within each group of multimers was calculated by multiplying the relative ratios of the low, intermediate or high MWMs with the total VWF:Ag level.

The ability of plasma from 10 ST and 10 AD patients with cirrhosis as well as 10 controls to support platelet adhesion was studied under flow conditions using reconstituted blood. ${ }^{30}$ Cellular elements of the reconstituted blood were isolated from healthy, O blood group donors. Adhesion study was completed within 2 hours after venipuncture. Platelet adhesion onto an uncoated polystyrene surface was carried out using an Impact- $\mathrm{R}$ cone and plate analyser (CPA; DiaMed, Switzerland). ${ }^{31}$ Platelet adhesion was quantified using acid phosphatase assay. ${ }^{32}$ Method details are the following. Citrated blood was centrifuged to obtain platelet-rich plasma (PRP, $150 \mathrm{~g}$, 10 minutes, $25^{\circ} \mathrm{C}$ ). Both the separated PRP and the remaining fraction of the blood were further centrifuged for platelet-poor plasma (PPP, 2,000 g, 10 minutes, $25^{\circ} \mathrm{C}$ ). After removing the PPP, the cellular parts were suspended in HEPES buffered saline, containing 0.0129 M sodium citrate, 0.005 M D-glucose and 40 $\mathrm{g} / \mathrm{L}$ bovine serum albumin (suspension buffer) to the original blood volume. Aliquots were pipetted into Eppendorf tubes for each adhesion experiment to obtain a platelet count of $200 \mathrm{~g} / \mathrm{L}$ and a haematocrit of 0.37 in a final volume of $0.14 \mathrm{~mL}$ reconstituted blood. After a washing step (centrifugation at $2,000 \times g, 10$ minutes, $25^{\circ} \mathrm{C}$ ), the supernatant was removed and the cells were suspended with the different cirrhotic or control plasmas to a final volume of $0.14 \mathrm{~mL}$. Reagents were purchased from Sigma-Aldrich, Germany. For the adhesion, $0.13 \mathrm{~mL}$ of this reconstituted blood was pipetted into the wells and submitted at a shear rate of $1,800 / \mathrm{s}$ for 2.5 minutes. Each well of the CPA was gently washed five times with PBS to remove unbound platelets and all compartments of the blood. Bound platelets were then lysed with $0.2 \mathrm{~mL}$ of lysis buffer containing acid phosphatase substrate: $0.1 \mathrm{M}$ citrate-phosphate buffer (pH 5.4), 0.1\% Triton X-100, $0.01 \mathrm{M}$ p-nitrophenol phosphate. Wells were incubated for 1 hour at $37^{\circ} \mathrm{C}$ in a humid box incubator and rotated continuously. Out of this mixture, $0.15 \mathrm{~mL}$ was added to $0.05 \mathrm{~mL} 2 \mathrm{~N} \mathrm{NaOH}$ into a 96-well plate to stop the enzyme reaction. The resultant colour was read at $405 / 545 \mathrm{~nm}$ and the number of the adhered platelets was calculated by the aid of the standard curve, which was constructed in parallel using the original platelet suspension. Intraassay coefficient of variation for the adhesion results was $12 \%$.

For the thrombin generation assay (TGA) citrated blood samples were centrifuged at $20^{\circ} \mathrm{C}$ for 15 minutes at 3,200 $\times g$ within 1 hour of venipuncture. Plasma was separated and centrifuged again $(3,200 \times g, 10$ minutes), and aliquots of the supernatant were stored at $-70^{\circ} \mathrm{C}$. Analysis was performed within 3 weeks. Individual aliquots were thawed in a $37^{\circ} \mathrm{C}$ water bath by tilting for 15 minutes, vortex mixed for 5 seconds and sampled immediately into black 96-well plates (Greiner GmbH, Germany). TGA were carried out using the Technothrombin TGA kit with fluorescent substrate and reagent $\mathrm{C}$ ( $50 \mathrm{pM}$ tissue factor $[\mathrm{TF}]$ and low concentration of phospholipid) according to the manufacturer's instructions. The reader, the plate with plasma and the reagent/substrate mixture were pre-heated to $37^{\circ} \mathrm{C}$, and then $0.06 \mathrm{~mL}$ reagent/ substrate mixture was added to $0.04 \mathrm{~mL}$ of plasma. The enzyme reaction was initiated by TF at a final concentration of $5 \mathrm{pM}$ and detected with a BIOTEK Flx 800 reader. Relative fluorescence units measured by the fluorimeter were converted into peak thrombin concentration (nM). A reference curve was constructed using a thrombin standard calibrated against the thrombin reference preparation of the WHO. The TGA kits, the Reader and the software were from Technoclone $\mathrm{GmbH}$.

\section{Ethical Considerations}

The study design was approved by the Regional and Institutional Research Ethics Committee (DEOEC RKEB/IKEB 53069/2011). Each patient was informed of the nature of this study and signed an informed consent.

\section{Statistical Analysis}

Variables were tested for normality using Shapiro-Wilk's W test. Continuous variables were summarized as medians and interquartile range (IQR; 25-75th percentiles). Categorical variables were compared with Fisher's exact test or chi-square test with Yates correction, as appropriate. Continuous variables were compared with Mann-Whitney $U$-test or Kruskal-Wallis $\mathrm{H}$ test with Dunn's multiple comparison post hoc analysis. The 
Spearman's non-parametric rank correlation test was used to determine correlations. To discriminate between survivors and non-survivors, the receiver operating characteristic (ROC) curve was used. AUC and corresponding 95\% confidence intervals (CIs) were calculated. Youden index was chosen to estimate the best discriminate threshold. Kaplan-Meier survival curves were plotted to estimate the cumulative probability of 6month survival of patients. For statistical analysis and graphical presentation, SPSS 24.0 (SPSS, Chicago, Illinois, United States) and GraphPad Prism 6 (San Diego, California, United States) programs were used. A two-sided probability value of $<0.05$ was considered to be statistically significant.

\section{Results}

\section{Baseline Characteristics}

Of 99 ST cirrhosis patients included, 51 had Child-Pugh A stage, 43 had Child-Pugh B and 5 had Child-Pugh C cirrhosis. At time of enrolment, 10 patients had stable chronic renal impairment, with creatinine level between 135 and $329 \mu \mathrm{mol} / \mathrm{L}$. AD events were defined as either bacterial infection, or HE, or sudden worsening of ascites or upper GI bleeding. The majority of the 54 AD patients had advanced cirrhosis, Child-Pugh B/C stage, but 6 patients had early Child-Pugh A cirrhosis. Thirty patients out of 54 had bacterial infection, with tense ascites in 17 patients, HE in 5 patients and upper GI bleeding in 5 patients. Out of the 24 patients without bacterial infection, 12 had upper GI bleeding (among them the 6 patients with Child-Pugh A stage), 8 had tense ascites and 6 had $\mathrm{HE}$ as the leading cause of $\mathrm{AD}$. Out of 54 AD patients, 28 had more than one event simultaneously. ACLF was detected only in 17 AD patients, grade 1 in 10 , grade 2 in 5 and grade 3 in 2 patients, respectively. Clinical characteristics of patients at inclusion are presented in - Table 1.

\section{VWF:Ag and Its Functional Activity Levels}

In cirrhosis, VWF:Ag, VWF:RCo level and VWF:CB activity was higher compared to healthy controls, see - Fig. 1A-C. Patients with $\mathrm{AD}$ had even higher values than patients with

Table 1 Epidemiologic, clinical and laboratory characteristics of patients with cirrhosis

\begin{tabular}{|c|c|c|c|c|}
\hline & & $\begin{array}{l}\text { Patients with } \\
\text { stable cirrhosis } \\
n=99\end{array}$ & $\begin{array}{l}\text { Patients with acute } \\
\text { decompensation } \\
n=54\end{array}$ & $p$-Value \\
\hline \multicolumn{2}{|c|}{ Gender (male/female) } & $47 / 52$ & $31 / 23$ & n.a. \\
\hline \multicolumn{2}{|l|}{ Age $(y)^{a}$} & $57(24-75)$ & $57(33-80)$ & 0.698 \\
\hline \multirow[t]{3}{*}{ Aetiology, $n(\%)$} & Alcohol & $63(63.6)$ & $43(79.6)$ & \multirow[t]{3}{*}{0.099} \\
\hline & Viral & $27(27.3)$ & $7(13.0)$ & \\
\hline & Other & $9(9.1)$ & $4(7.4)$ & \\
\hline \multirow[t]{2}{*}{ Child-Pugh stage, $n$} & $\begin{array}{l}\text { A } \\
B\end{array}$ & $\begin{array}{l}51(51.5) \\
43(43.4)\end{array}$ & $\begin{array}{l}6(11.1) \\
24(44.4)\end{array}$ & \multirow[t]{2}{*}{$<0.001$} \\
\hline & $\mathrm{C}$ & $5(5.1)$ & $24(44.4)$ & \\
\hline \multicolumn{2}{|l|}{ MELD score } & $12(9-14)$ & $16(12-23)$ & $<0.001$ \\
\hline \multicolumn{2}{|c|}{ White blood cell count $(\mathrm{g} / \mathrm{L})^{\mathrm{a}}$} & $5.3(4.2-6.8)$ & $7.1(4.6-11.0)$ & 0.003 \\
\hline \multicolumn{2}{|l|}{ Platelet count $(\mathrm{g} / \mathrm{L})^{\mathrm{a}}$} & $112(70-161)$ & $108(71-165)$ & 0.924 \\
\hline \multicolumn{2}{|c|}{ Serum sodium $(\mathrm{mmol} / \mathrm{L})^{a}$} & $141(138-143)$ & $138(134-141)$ & 0.001 \\
\hline \multicolumn{2}{|c|}{ Serum creatinine $(\mu \mathrm{mol} / \mathrm{L})^{\mathrm{a}}$} & $64(54-84)$ & $75(50-133)$ & 0.122 \\
\hline \multicolumn{2}{|c|}{ C-reactive protein $(\mathrm{mg} / \mathrm{L})^{a}$} & $3.8(1.5-9.0)$ & $18.1(6.0-48.5)$ & $<0.001$ \\
\hline \multicolumn{2}{|c|}{ Serum bilirubin $(\mu \mathrm{mol} / \mathrm{L})^{a}$} & $31(19-50)$ & $61(31-179)$ & $<0.001$ \\
\hline \multicolumn{2}{|c|}{ Aspartate aminotransferase $(\mathrm{U} / \mathrm{L})^{\mathrm{a}}$} & $51(33-83)$ & $67(45-125)$ & 0.002 \\
\hline \multicolumn{2}{|c|}{ Alanine aminotransferase $(\mathrm{U} / \mathrm{L})^{\mathrm{a}}$} & $33(21-57)$ & $31(21-58)$ & 0.887 \\
\hline \multicolumn{2}{|l|}{ Serum albumin $(\mathrm{g} / \mathrm{L})^{\mathrm{a}}$} & $37(33-41)$ & $28.5(24-32)$ & $<0.001$ \\
\hline \multicolumn{2}{|l|}{$I^{\prime N R}{ }^{a}$} & $1.1(1.2-1.3)$ & $1.4(1.2-1.7)$ & $<0.001$ \\
\hline \multicolumn{2}{|l|}{ Ascites present, $n(\%)$} & $41(41.4)$ & $38(70.0)$ & 0.001 \\
\hline \multirow{4}{*}{$\begin{array}{l}\text { Patients with acute } \\
\text { decompensation, } \\
n(\%)\end{array}$} & Tense ascites formation & & $25(46.3)$ & n.a. \\
\hline & Upper GI bleeding & & $17(31.5)$ & n.a. \\
\hline & $\mathrm{HE}$ & & $11(20.4)$ & n.a. \\
\hline & Bacterial infection & & $30(55.6)$ & n.a. \\
\hline \multicolumn{2}{|c|}{ Six-month mortality, $n(\%)$} & $4(4.0)$ & $17(31.5)$ & $<0.001$ \\
\hline
\end{tabular}

Abbreviations: $\mathrm{Gl}$, gastrointestinal; HE, hepatic encephalopathy; INR, international normalized ratio; MELD, model for end-stage liver disease; n.a., not applicable.

${ }^{a}$ Median, interquartile range. 
A

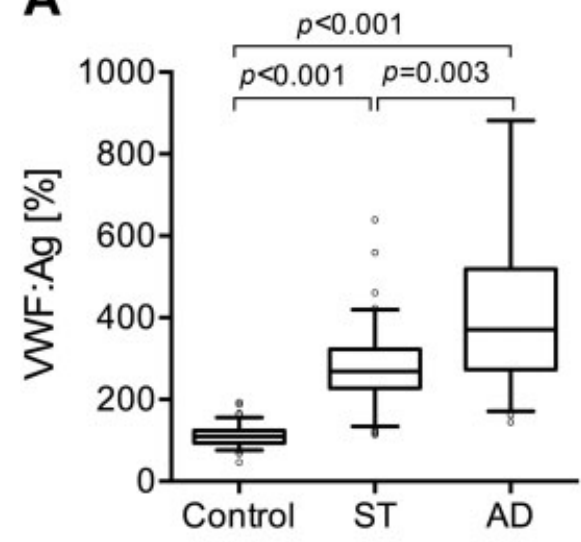

B
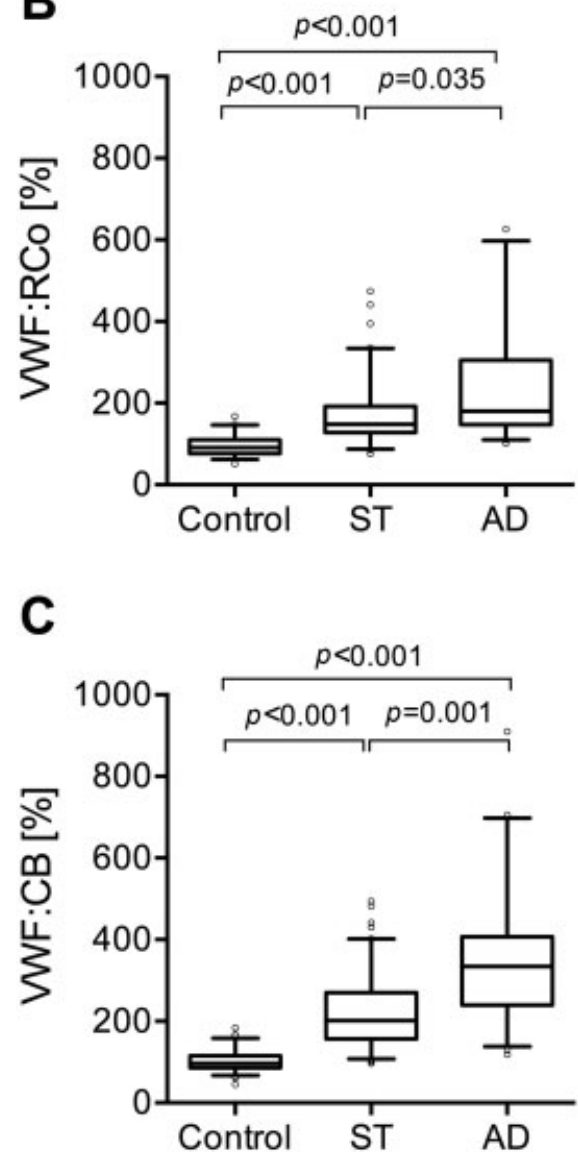

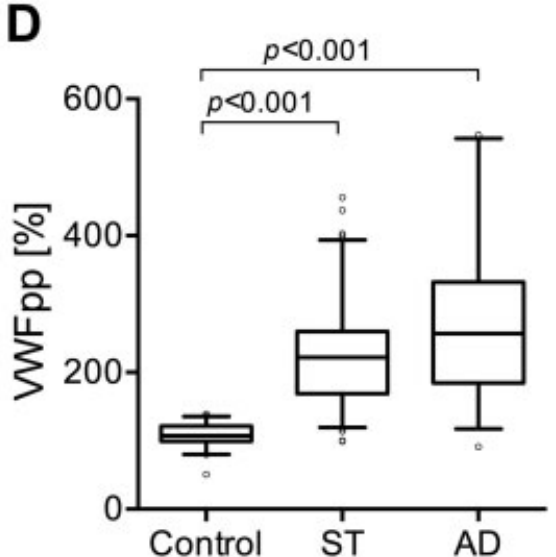

E

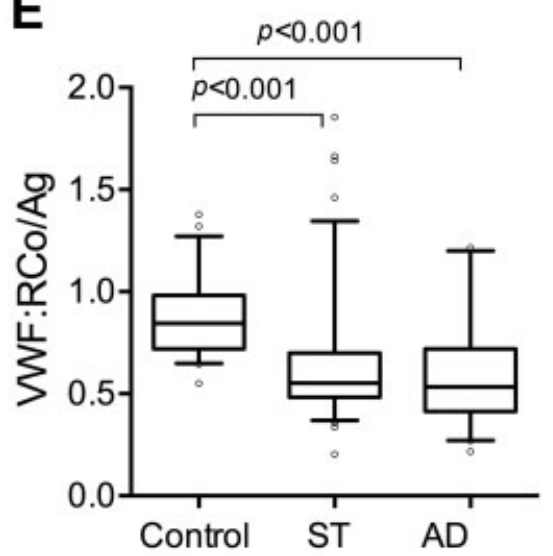

$\mathbf{F}$

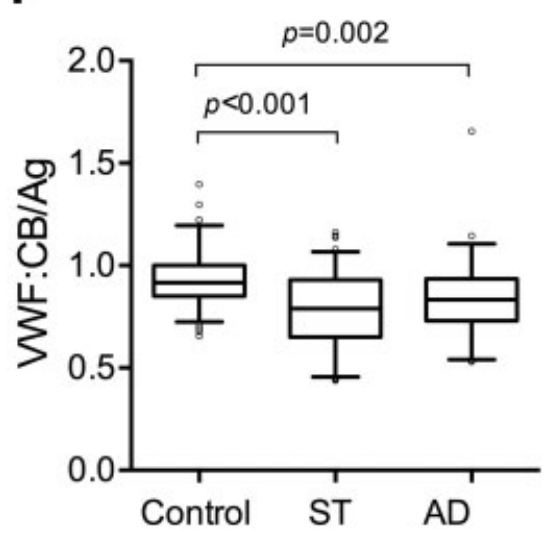

Fig. 1 Plasma von Willebrand factor antigen (VWF:Ag), pro-peptide (VWFpp) and VWF functional activities in cirrhosis. VWF:Ag (A), VWF ristocetin co-factor activity (VWF:RCo) (B), VWF collagen-binding activity (VWF:CB) (C), VWFpp (D), VWF:RCo/VWF:Ag (E) and VWF:CB/VWF:Ag (F) in plasma of healthy controls and patients with stable (ST) cirrhosis and acute decompensation (AD) are shown. VWF:Ag, VWFpp and VWF activity levels are expressed as percentage of pooled normal plasma. Lines denote median values, boxes represent 25 th to 75 th percentiles and whiskers indicate the 5th to 95th range. $p$-Values were calculated by Kruskal-Wallis $\mathrm{H}$ test with Dunn's multiple comparison post hoc analysis.

ST (VWF:Ag \% AD vs. ST: 371 [275-513] vs. 269 [228-323], $p=0.003$; VWF:RCo \% AD vs. ST: 180 [148-294] vs. 148 [128$192], p=0.035$ and VWF:CB AD vs. ST: 335 [245-405] vs. 201 [156-269], $p=0.001$ ).

Although both types of functional activities of VWF were elevated in each group of patients, they did not reach the level of VWF:Ag. The VWF:RCo/VWF:Ag ratio in the control group was $0.84(0.72-0.98)$, whereas it was lower in both cirrhotic groups. In ST patients 0.55 (0.48-0.70) and in AD patients 0.53 (0.41-0.72) was found (-Fig. 1E). The VWF:CB/ VWF:Ag ratio was also reduced in both patient groups with cirrhosis. In ST patients 0.79 (0.65-0.93) and in AD patients $0.83(0.73-0.93)$, whereas in controls $0.92(0.85-1.00)$ could be obtained (-Fig. 1F).

A moderate correlation was found between VWF:Ag and VWF:RCo $(r=0.521, p<0.001)$, and strong correlation 
between VWF:Ag and VWF:CB $(r=0.835, p<0.001)$ as well as VWF:CB and VWF:RCo $(r=0.619, p$ 0.001) in cirrhosis.

In both cirrhotic groups, the level of VWFpp (-Fig. 1D) was higher as compared to the controls. In the ST group, 222 (169-260)\%, in AD patients 257 (196-323)\%, while in the controls 108 (99-121)\% levels were found.

VWF:Ag levels within the AD group significantly differed between patients with (475 [355-649]\%) and without (350 [266-446]\%) ACLF $(p=0.013)$.

\section{Analysis of VWF Multimers}

SDS agarose electrophoretic pattern of VWF and the corresponding densitometric curves of plasma samples from patients with ST or AD disease and controls are shown in -Fig 2. According to the visual analysis (-Fig. 2A and -Supplementary Fig. S1, available in the online version), the presence of ULMWM could be identified in 39 (25.5\%) patients with cirrhosis. The majority of them was in the AD group (25 out of 54 patients, 46.3\%), while 14/99 (14\%) were found in the ST group. Computerized analysis of the

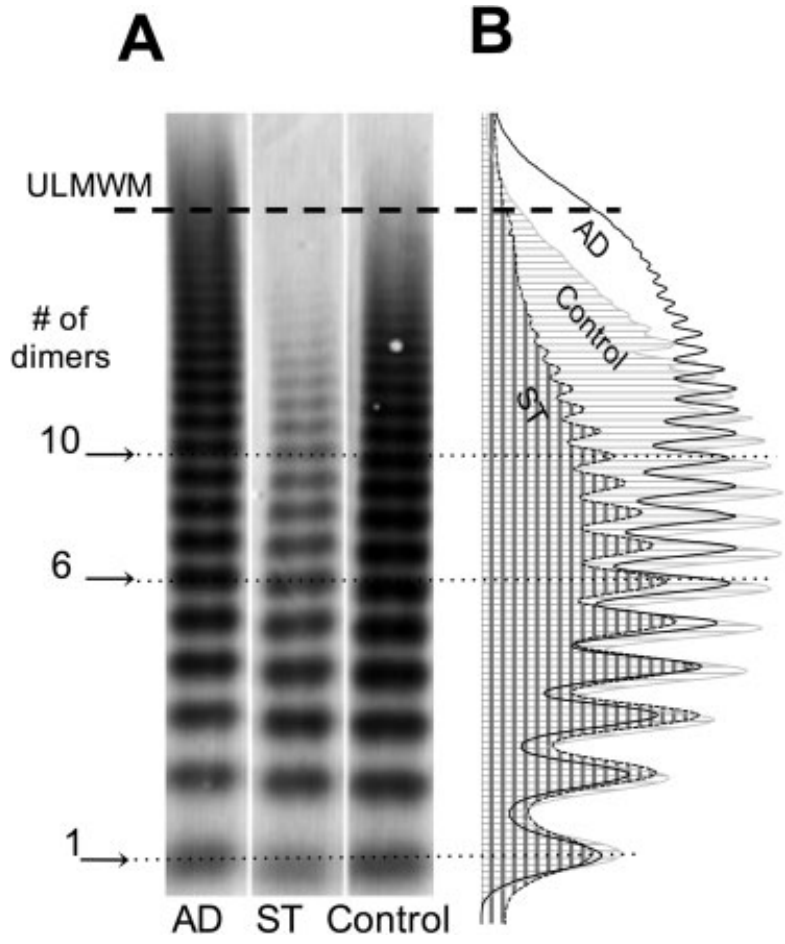

Fig. 2 Multimeric pattern of von Willebrand factor (VWF) after separation on $0.8 \%$ agarose gel and the corresponding densitometric curves. (A) Characteristic plasma sample of a patient with acute decompensation (AD), stable (ST) cirrhosis and healthy control. The presence of ultra-large molecular weight multimers (ULMWM) is seen in the sample of the AD patient. No ULMWM can be seen in the sample of ST cirrhosis or healthy control. The dotted lines across the lanes represent critical separation on the distribution ladder according to the number of the corresponding bands. The critical separation lines: $\# 1=1$ dimer; \#6 = 6 dimers and \#10 = 10 dimers. Dimers $<6$ are defined as low molecular weight multimers (LMWM), dimers between 6 and 10 defined as intermediate molecular weight multimers (IMWM) and dimers > 10 defined as high molecular weight multimers (HMWM) including ULMWM. (B) The corresponding densitometric curves indicated with different lines and areas as marked at the bottom of $\mathrm{A}$. densitometric curves ( $\mathbf{- F i g . ~ 2 B}$ ) was next performed, and from the total AUC, the relative ratios of the LMWM, IMWM and HMWM were determined, respectively. We further analysed the samples according to the presence of ULMWM. The ratios of the LMWMs are shown in - Fig. 3A. It was 0.28 (0.25-0.31) in controls and the ratios were higher in both ST and $\mathrm{AD}$ patients with or without ULMWM. In the samples without ULMWM, we measured $0.38(0.35-0.42)$ and 0.39 (0.32-0.46) LMWMs, for ST and AD patients, respectively. In patients with ULMWM, the proportion of LMWM decreased to $0.32(0.26-0.36)$ in ST and $0.32(0.29-0.36)$ in AD patients but both ratios remained higher than the controls $(p=0.018$ and $p<0.001$, respectively). The proportions of the IMWM (-Fig. 3B) did not show any difference between the ST group and the controls. However, in patients with $\mathrm{AD}$, both with and without ULMWM, the ratios were significantly lower as compared to either controls or ST. Analysing the multimer proportions within the HMWM fraction (-Fig. 3C), we observed $0.4(0.37-0.44)$ in controls, whereas we detected only $0.30(0.25-0.34)$ and $0.29(0.24-0.39)$ in patients without ULMWM in ST and AD patients, respectively. Remarkably, in patients with ST as well as AD in the presence of ULMWM the ratios of HMWM were higher and became similar to controls, 0.37 (0.31-0.43) and 0.40 (0.32-0.44), respectively.

The absolute amounts of VWF measured within the different fractions of the multimers are shown in - Fig. 3D and $\mathbf{E}$. We would like to emphasize that within the HMWM group in the presence of ULMWM a significantly higher quantity of VWF:Ag was detected in both ST and AD patients.

\section{ADAMTS13 Antigen and Activity Levels}

In patients with $\mathrm{AD}$, ADAMTS13 activity level was decreased compared to ST and healthy controls (57 [29-94] vs. 98 [67-132] and 106 [67-117]\%, $p<0.001$ for both).

Similarly, low ADAMTS13 antigen levels were detected in patients with AD compared to ST and controls (66 [43-101] vs. 120 [89-155] and 101 [91-115]\%, respectively, $p<0.001$ for both).

We further analysed the ADAMTS13 activity and antigen levels, according to the presence of ULMWM within both the cirrhotic groups (-Fig. 4). In patients without ULMWM, ADAMTS13 activity levels were not different from controls, in ST 104 (71-137)\% and in AD 91 (60-110)\% were found. In patients with ULMWM, the values were significantly lower as compared to controls and those without ULMWM. In ST 65 (51-99)\% and in AD 33 (24-49)\% were measured. Similar alterations were observed for the ADAMTS13 antigen levels, except, that in ST patients without ULMWM, the level was higher than in the controls. In both patient groups with ULMWM, the antigen levels were significantly lower, than in patients without ULMWM. In the AD group, ADAMTS13 activity level in the presence or absence of ACLF was 49 (29-68)\% and 70 (44-107)\%, respectively ( $p=0.171)$.

We also analysed the correlation between the quantity of VWF:Ag calculated within the HMWM fraction and ADAMTS13 activity in ST and AD cirrhotic patients as well as in controls. As is shown in - Fig. 5, a negative correlation (Spearman's $r=-0.43, p=0.01$ ) was found between these 
A

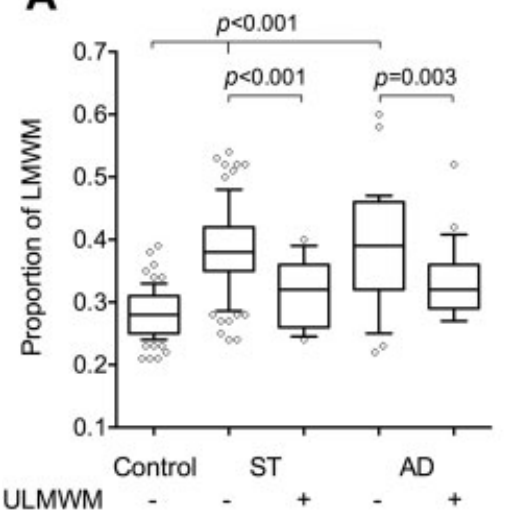

D

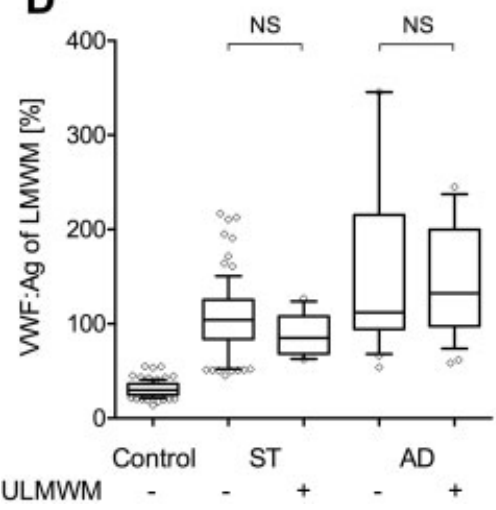

B

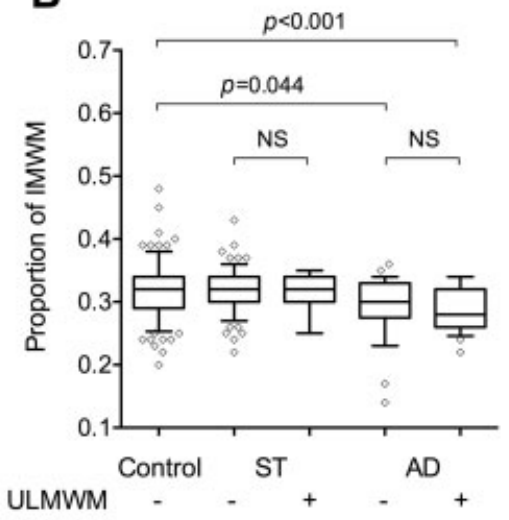

E

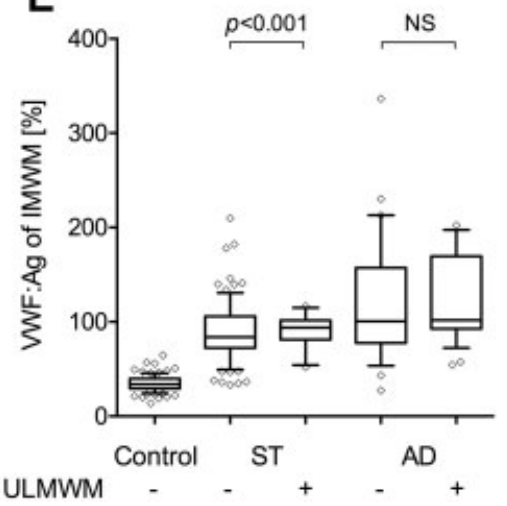

C
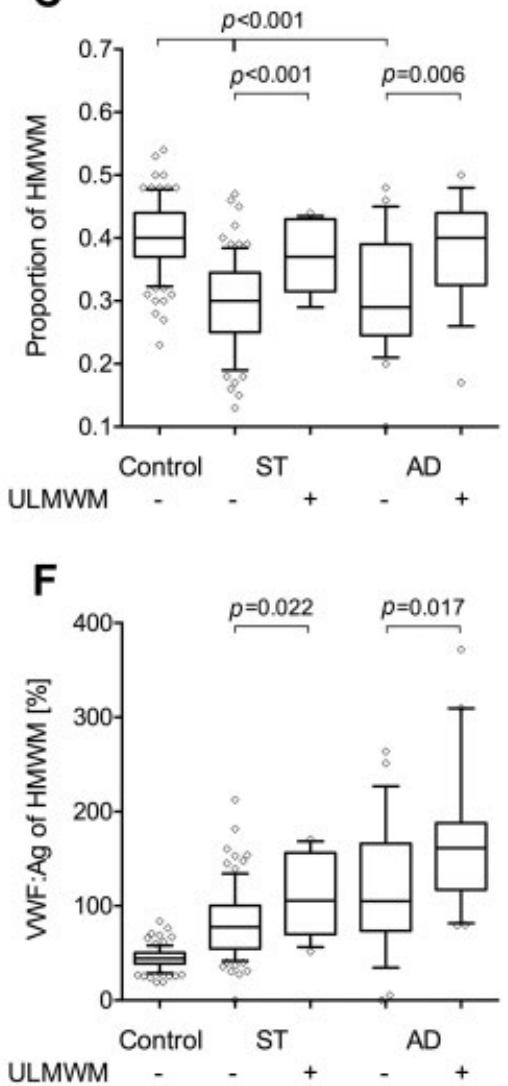

Fig. 3 Proportions of the low molecular weight multimers (LMWM), intermediate molecular weight multimers (IMWM) and high molecular weight multimers (HMWM) groups of bands and the corresponding absolute amount of von Willebrand factor (VWF) in each group of multimers. (A) The proportion of the area under the curve (AUC) of the first 5 bands considered as LMWM (1-5 dimers). (B) The proportion of the AUC of bands between 6 and 10 named as IMWM (6-10 dimers). (C) The proportion of the AUC of larger than 10 multimers, considered as HMWM including the area where VWF did not resolve into bands anymore (> 10 dimers, HMWM). (D-F) The absolute amount of VWF within the LMWM, IMWM and HMWM groups of multimers. The group of stable (ST) and acute decompensation (AD) patients are separated according to the presence or absence of ultra-large molecular weight multimers (ULMWM). Lines denote median values, boxes represent 25th to 75 th percentiles and whiskers indicate the 5th to 95th range. $\mathrm{p}$-Values were calculated by Kruskal-Wallis $\mathrm{H}$ test with Dunn's multiple comparison post hoc analysis.

A

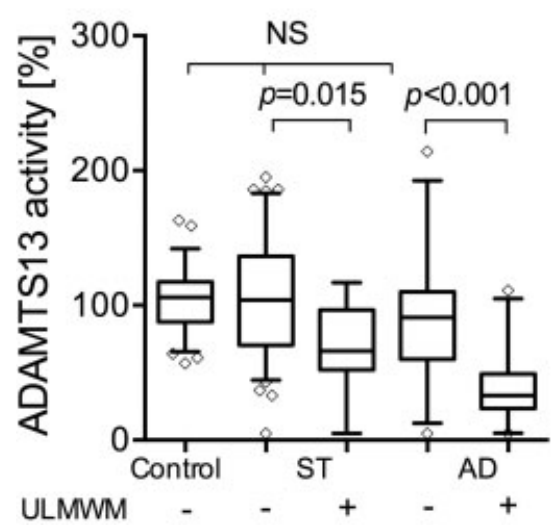

B

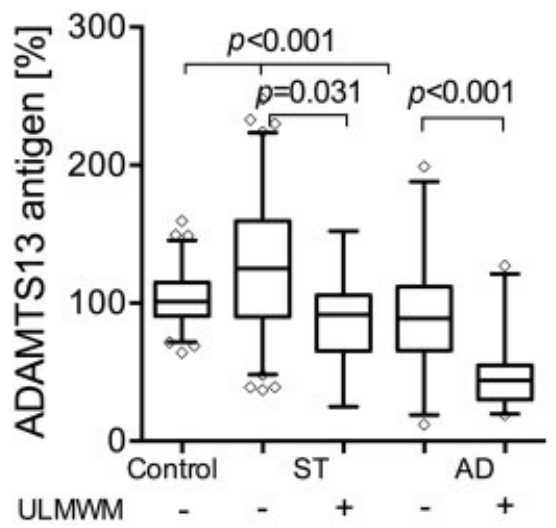

Fig. 4 Plasma a disintegrin-like and metalloproteinase with thrombospondin type-1 motifs $\underline{13}$ (ADAMTS13) activity (A) and antigen level (B) in plasma of healthy controls and patients with stable (ST) cirrhosis and acute decompensation (AD) separated according to the presence of ultralarge molecular weight multimers (ULMWM). ADAMTS13 activity and antigen levels are expressed as percentage of pooled normal plasma. Lines denote median values, boxes represent 25th to 75 th percentiles and whiskers indicate the 5 th to 95 th range. $p$-Values were calculated by Kruskal-Wallis H test with Dunn's multiple comparison post hoc analysis. 
A

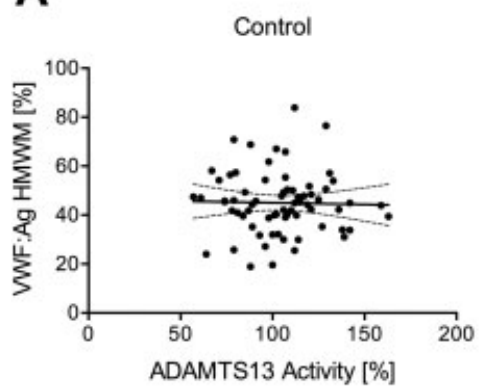

B

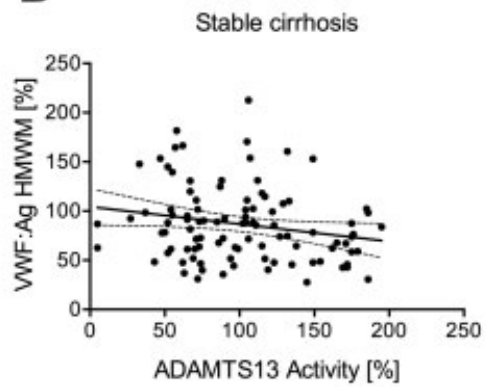

C

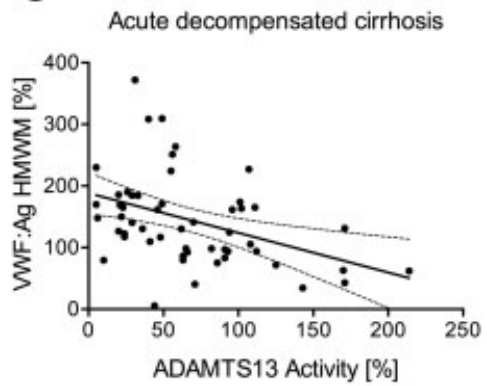

Fig. 5 Linear regression line and correlation between the quantity of von Willebrand factor (VWF) high molecular weight multimers (HMWM) and a disintegrin-like and metalloproteinase with thrombospondin type-1 motifs 13 (ADAMTS13) activity. HMWM VWF antigen (VWF:Ag) levels were plotted versus the plasma ADAMTS13 activities of healthy controls (A), Spearman's $r=-0.043, p=0.722, n=70$; stable cirrhosis patients (B), Spearman's $r=-0.18, p=0.073, n=98$; and patients with acute decompensation (C), Spearman's $r=-0.43, p=0.01, n=54$.

parameters in AD patients, that is, the lower the ADAMTS13 activity was, the higher the VWF:Ag level was found in the HMWM fraction. However, in ST patients (Spearman's $r=$ $-0.18, p=0.073$ ), as well as in the controls there was no significant correlation (Spearman's $r=-0.043, p=0.722$ ).

We then investigated the association between ADAMTS13 activity and systemic inflammation, represented by CRP, in all AD patients. Patients with ADAMTS13 activity less than 50\% (calculated by ROC analysis and Youden index), had higher CRP levels compared to those with ADAMTS13 activity above 50\% (29.8 [6.6-97.4] vs. 10.9 [7.1-34.5] $\mathrm{mg} / \mathrm{L} ; p=0.048$ ). In line with this, in AD patients with ULMWM the CRP level was higher as compared to the ones without ULMWM (22.96 [7.1$83.6]$ vs. 10.9 [4.8-36.8] $\mathrm{mg} / \mathrm{L} ; p=0.078)$.

\section{Platelet Adhesion Assay}

As shown in - Fig. 6A, adhesion of normal platelets showed a stepwise increase in the presence of cirrhotic plasmas, reaching the highest level in AD patients with ULMWM. VWF:Ag level was 106 (86-120)\% in controls, 309 (279-327)\% in ST and $356(265-384) \%$ in AD patients. VWF:Ag levels in the absence $(n=14)$ or presence $(n=6)$ of ULMWM in cirrhotic patients, were 305 (261-348)\% and 340 (298-384)\%, respectively. The VWF:Ag levels were not significantly different.

\section{Thrombin Generation Assay}

As shown in -Fig. 6B, peak thrombin concentration was increased in both cirrhotic groups as compared to controls. We measured $192(158-238) n M$ in controls $(n=31), 271$

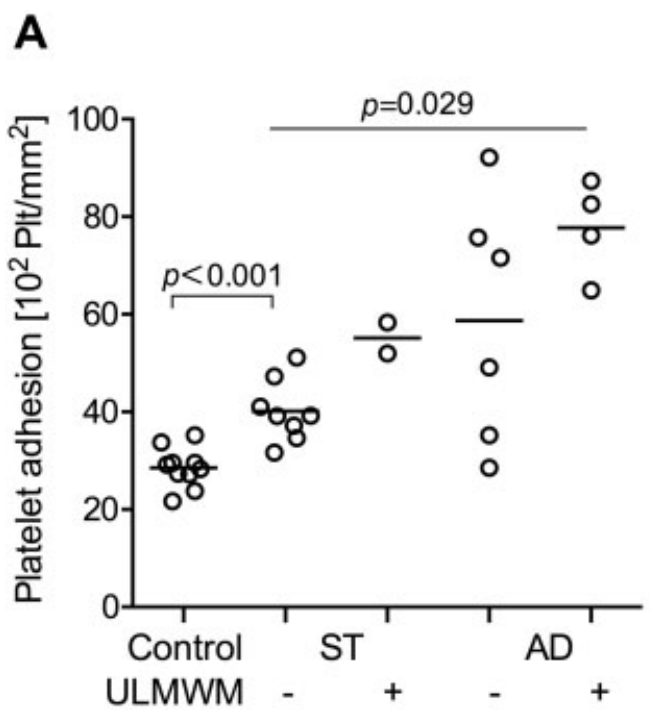

B

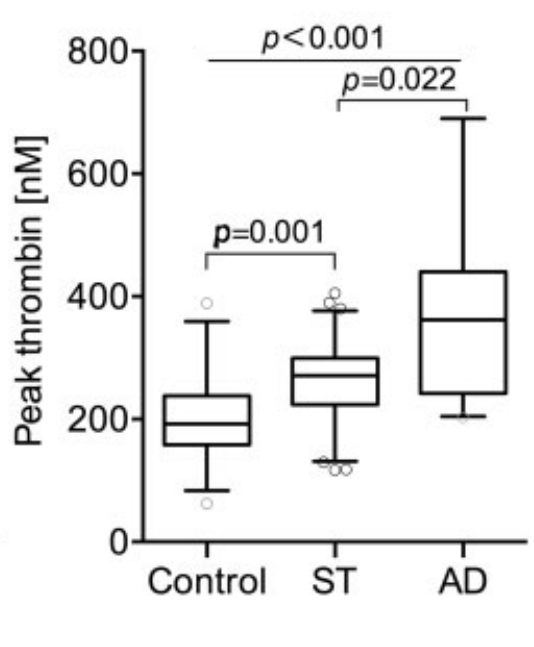

Fig. 6 Prothrombotic tendency in patients with stable (ST) cirrhosis and acute decompensation (AD). (A) Adhesion of normal platelets in the presence of plasma of healthy controls or patients. Platelet adhesion activity of plasma from patients with cirrhosis was studied using reconstituted blood at 1,800/s, 2.5 minutes using a cone and plate analyser with uncoated wells (DiaMed Impact-R). Ten ST, 10 AD patients and 10 controls were selected. The number of platelets (PIt) adhering were determined by acid phosphatase activity. Results of cirrhotic samples are separated according to the absence or presence of ultra-large molecular weight multimers (ULMWM). Lines denote median values. $p$-Values were calculated by Mann-Whitney and Kruskal-Wallis $\mathrm{H}$ tests. (B) Thrombin generation in plasma of controls and both cirrhotic patient groups. Samples from 31 controls, 62 ST and 27 AD cirrhotic patients were analysed using the Technothrombin kit and results are expressed as peak thrombin concentration. Lines denote median values, boxes represent 25th to 75th percentiles and whiskers indicate the 5th to 95 th range. p-Values were calculated by Kruskal-Wallis $\mathrm{H}$ test with Dunn's multiple comparison post hoc analysis. 
$(223-293) \mathrm{nM}$ in ST $(n=62)$ and $362(242-440) \mathrm{nM}$ in AD $(n=27)$ patients.

\section{Association of VWF:CB, ADAMTS13 Activity and CRP with Survival}

Within the ST group 4 out of 99 patients, whereas in the AD group 17 out of 54 patients died. The causes of death were liver-related in all patients, namely, GI bleeding in 3 patients, infection in 11 patients and hepatorenal syndrome in 7 patients. The areas under the ROC curves to predict 6 -month survival (-Fig. 7A) were 0.708 (95\% CI, 0.629-0.778) for VWF: Ag level, 0.717 (95\% CI, 0.639-0.787) for VWF:CB level and 0.612 (95\% CI, 0.530-0.6 90) for ADAMTS13 activity. The optimum cut-offs for predicting poor 6-month survival according to the Youden index were VWF:CB activity > 245\% with $80.95 \%$ sensitivity and $58.33 \%$ specificity and ADAMTS13 activity $<50 \%$ with $47.62 \%$ sensitivity and $82.44 \%$ specificity. Patients with either $>245 \%$ VWF:CB activity, or $<50 \%$ ADAMTS13 activity or $>10 \mathrm{mg} / \mathrm{L}$ CRP level had significantly poorer 6 -month survival. The 6 -month survivals were $74.6 \%$ versus $94.4 \%$ in patients according to the VWF:CB activity (-Fig. 7B), 69.2\% versus $89.5 \%$ according to the ADAMTS13 activity (- Fig. 7 C) and $68.9 \%$ versus $92.3 \%$ according to the CRP level (-Fig. 7D). Within the AD group, 6-month survivals were $60.1 \%$ versus $80.8 \%$ for VWF:CB activity, $57.2 \%$ versus $72.0 \%$ according to the ADAMTS13 activity and $55.7 \%$ versus $78.6 \%$ for CRP levels. Within the AD group, these survivals showed high numerical difference, but due to the low number of patients, these data did not reach statistical significance.

\section{Discussion}

In this study, we performed detailed analyses of both VWF and ADAMTS13 in one of the largest group of patients with cirrhosis, published so far. We have tried to clarify hitherto unanswered questions in our study. Namely, (1) what is the effect of $A D$ and systemic inflammation in patients with cirrhosis on VWF parameters including multimer distribution as well as ADAMTS13 activity as compared to patients with stable cirrhosis; and (2) can the presence of a prothrombotic state be confirmed in these patients?

It is known that VWF:Ag levels are highly elevated in patients with cirrhosis. ${ }^{33,34}$ Several previously published data provide strong evidence that the level of VWF:Ag is related to the severity of the disease, reaching the highest levels in Child C patients. The platelet glycoprotein Ib binding
A
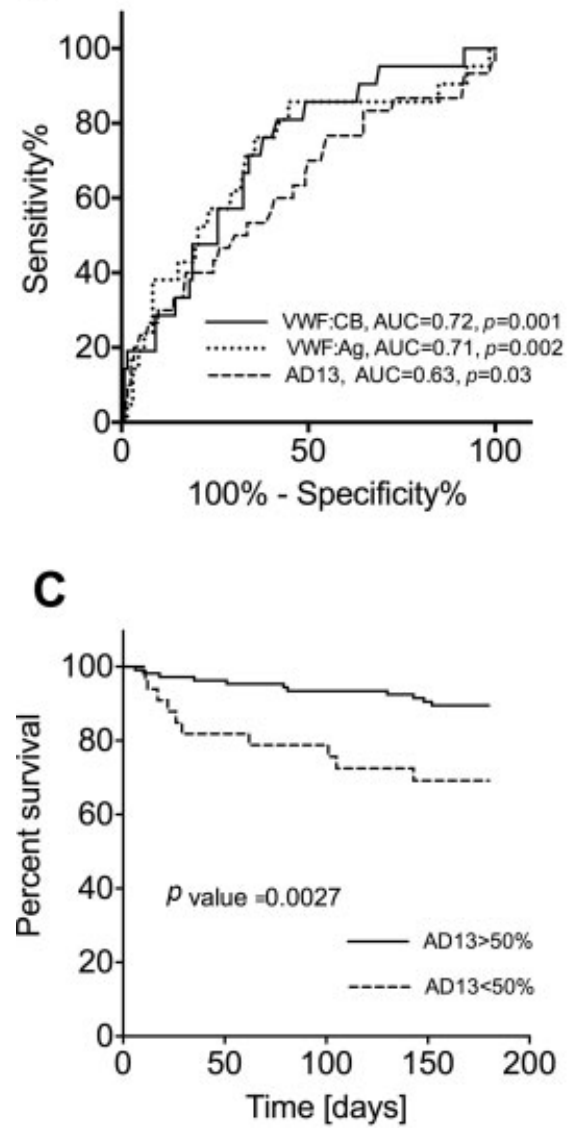

B

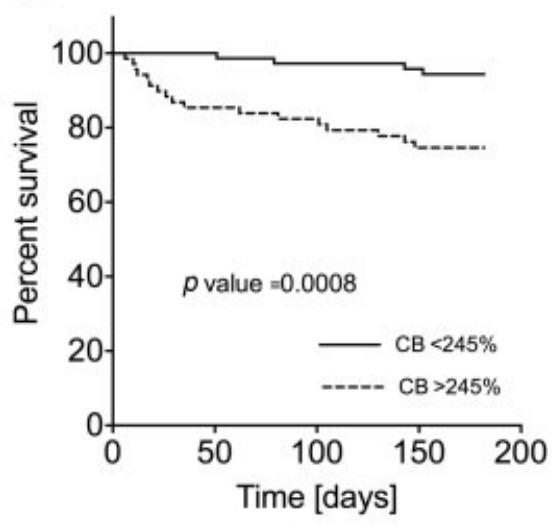

D

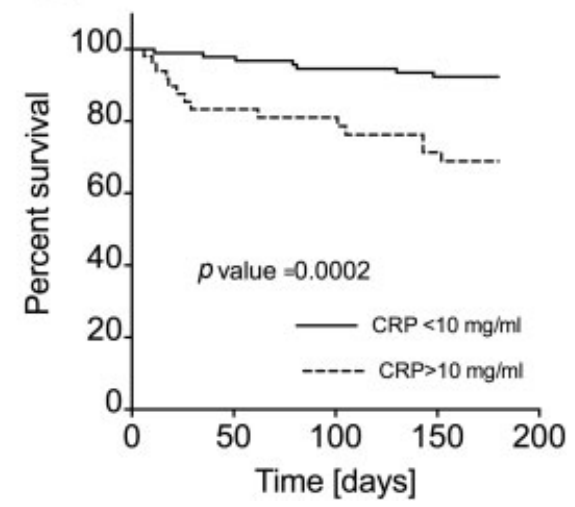

Fig. 7 Survival of cirrhotic patients. Receiver operating characteristics curves for prediction of 6-month survival for von Willebrand factor antigen (VWF:Ag) (dotted line), VWF collagen-binding activity (VWF:CB) (solid line) and a disintegrin-like and metalloproteinase with

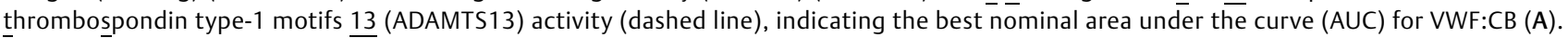
Kaplan-Meier curves indicate higher 6-month survival for patients with $<245 \%$ VWF:CB (B), or with $>50 \%$ ADAMTS13 activity (C) or $<10$ mg/L C-reactive protein (CRP) level (D). 
functional capacity of VWF, a feature that is measured by VWF:RCo, is also substantially elevated, albeit not to the same extent as the VWF:Ag level. ${ }^{3,35}$

We could demonstrate that VWF:CB activity, which measures the binding capacity of VWF to collagen, was even higher than the VWF:RCo as we show here for the first time. Our finding is in contrast to the previous publications of Lisman's group. As they observed, VWF:CB was lower in any type of liver diseases than in controls, $3,36,37$ despite that this activity is more sensitive to the presence of high multimers than the VWF: $\mathrm{RCo}^{38}$ There was, however, a major difference between their and the methodology that we and others are using. Our VWF:CB measurements were done in parallel to the VWF:Ag measurements from the same sample dilutions. Lisman et al, however, added the same amount of VWF:Ag into the wells (5\% and 2.5\% of the amount of VWF present in pooled normal plasma) for the determination of the VWF: $\mathrm{CB}$, hence their data better reflect the VWF:CB/VWF:Ag ratio, which also in our hands was reduced. Moreover, they demonstrated that plasma from patients either with cirrhosis or acute liver failure induced an increased platelet adhesion to collagen surface, which definitively suggests a wellfunctioning VWF:CB activity. These adhesion results under flow conditions could further confirm our findings.

The relative decrease of VWF:RCo and VWF:CB suggests a significant qualitative alteration of the multimeric structure of VWF. Indeed, we could demonstrate two major types of changes in the multimeric structure of VWF. In the majority of cirrhotic patients, where ULMWM was not present, the relative ratio of the LMWM fraction was increased, while the ratio of the haemostatically most active HMWM was reduced, that is, a shift from HMWM to LMWM could be observed. Remarkably, the absolute quantity of VWF within LMWM showed a threefold and in the HMWM only a twofold increase as compared to controls. Another major change could be seen in almost $50 \%$ of the AD patients where the presence of ULMWM of VWF could be detected. In these patients, the relative ratio as well as the absolute quantity of VWF:Ag within the HMWM fraction was much higher than in AD patients without ULMWM. Furthermore, we measured both lower activity and antigen levels of the VWF-cleaving protease ADAMTS13. In contrast, in patients without ULMWM, ADAMTS13 levels were similar to controls. Hence, in the group of patients with advanced liver cirrhosis, we suggest that probably the primary step is a decrease in ADAMTS13, which might be the consequence of the AD event combined with the systemic inflammation, represented by the higher CRP. Within the AD patient group ACLF represents an even more severe disease, in this minor group we found the highest VWF:Ag level and ADAMTS13 was also low. These findings are in line with previous results. ${ }^{39}$

We put forward for consideration on the one hand, an ADAMTS13 independent alteration of VWF in those mostly stable cirrhotic patients in whom ADAMTS13 level is normal and ULMWM are not present. In these patients, degradation of VWF multimers may be due to other proteolytic enzymes, like plasmin ${ }^{40}$ or other serine proteases. ${ }^{41}$ On the other hand, in the most advanced cirrhotic patients ULMWM can be detected due to the low level of ADAMTS13. The significantly increased VWF activity in cirrhotic plasmas containing ULMWM was confirmed by our platelet adhesion experiment. Our results suggest that indeed in the most severe cirrhotic patients a pro-thrombotic state can develop, to some extent reminiscent to what happens in TTP. ${ }^{8}$ Infections as well as systemic inflammation are supposed to induce a pro-thrombotic tendency in cirrhotic patients. This was proved by several lines of evidence, such as elevation of coagulation factor VIII or VWF levels and platelet activation due to gut-derived endotoxins; or demonstration of increased thrombin generation. ${ }^{16,18,39,42-45}$ Our observations are in line with these previous studies, since we could demonstrate increased platelet adhesion together with increased thrombin generation in half of our AD patients.

ADAMTS13 is secreted by hepatic stellate cells, ${ }^{10}$ which are known to be activated in cirrhotic patients. ${ }^{11}$ We speculate that in AD the synthesis of ADAMTS13 is reduced, since inflammatory cytokines are known to inhibit ADAMTS13 synthesis by the stellate cells. ${ }^{46}$ Furthermore, as the half-life of ADAMTS13 is about 2 to 3 days, ${ }^{47}$ the ULMWM can appear within a couple of days. In patients with $A D$, the very low ADAMTS13 activity together with the presence of ULMWM might represent an already significantly advanced disease stage. From these data, we can propose that multimeric analysis of VWF and ADAMTS13 activity measurement, albeit that they are not routine tests, could help to identify patients with a pro-thrombotic tendency. As standardized ADAMTS13 activity measurement, a WHO international standard and quality controls are available, we propose to measure ADAMTS13 activity especially in those centres where care is taken of the most advanced patients.

The severity of these events is clearly demonstrated by the poor 6-month survivals of these patients. Higher VWF:CB activity ( $>245 \%$ ), higher CRP ( $>10 \mathrm{mg} / \mathrm{L}$ ) as well as low ADAMTS13 activity $(<50 \%)$ either in the total group of patients or within the patients with $A D$ resulted in higher mortality. Systemic inflammation together with the activation of the haemostatic system can play a role in the disease progression and this raises the need for thromboprophylaxis in these patients. Villa et al could demonstrate that low molecular weight heparin did prevent portal vein thrombosis and delayed hepatic decompensation. ${ }^{43}$ No data are available at present for antiplatelet prophylaxis.

There are some controversies on the multimeric structure of VWF as well as ADAMTS13 levels in cirrhotic patients categorized by the Child-Pugh system. Lisman et al suggested that there are less HMWM compared to controls and very variable ADAMTS13 levels. ${ }^{3}$ Uemura et al were the only ones who categorized the patients according to three different multimeric patterns, ${ }^{35}$ and LMWM dominated in the first group. In the second group, normal multimeric structure was demonstrated, whereas in the third one, the presence of ULMWM was detected. They showed a negative correlation between ADAMTS13 levels and the severity of cirrhosis and suggested an increased intra-hepatic formation of micro-thrombi. However, more than $50 \%$ of the Japanese patients (57/109) suffered also from hepatocellular carcinoma which significantly increased the heterogeneity of their patients. In another study, 
Reuken et al investigated the effect of systemic inflammation, mostly due to bacterial infections, on VWF. ${ }^{44}$ Both VWF levels as well as ADAMTS13 levels were very similar to our findings, but they could not demonstrate any change in the multimeric structure of VWF. They used, however, throughout their study, $1.6 \%$ SDS-agarose gel electrophoresis. As a consequence, the migration of the large multimers from the stacking gel into the separating gel was most probably less complete than into the 0.8 or $0.65 \%$ agarose gels we used, which therefore seems to be more sensitive to identify the changes of the multimeric structure of VWF, especially the ULMWM.

In summary, we here for the first time demonstrate, that VWF:CB activity measurement has similar value as VWF:RCo determination in cirrhosis. Also, we could quantitatively demonstrate that the ratio of the LMWM was significantly increased in stable cirrhotic patients. Furthermore, in the most severe cirrhotic patients with AD significantly reduced ADAMTS13 activity could be found, along with the presence of ULMWM, which are markers of thrombotic micro-angiopathy and possible contributors to the disease progression.

\section{What is known about this topic?}

- High von Willebrand factor (VWF) level and ristocetin cofactor activity are present in cirrhosis that counterbalance low platelet count.

- ADAMTS13 activity is low in advanced cirrhosis.

- There are controversies on the multimeric structure of VWF in cirrhosis.

\section{What does this paper add?}

- Collagen-binding activity was similarly elevated as ristocetin co-factor activity and was strongly correlated with VWF antigen in cirrhosis.

- The ratio of low molecular weight VWF multimers increased and that of the high multimers decreased in stable cirrhosis.

- Ultra-large VWF multimers and the lowest ADAMTS13 activity together with increased thrombin generation were evidenced in patients with acute decompensation resulting in increased pro-thrombotic activity.

\section{Funding}

This work was supported by a research grant of the National Research Development and Innovation Office (NVKP-16-1-2016-0017).

\section{Conflict of Interest}

None.

\section{Acknowledgement}

The authors are very grateful to Eva Tomori and Maria Fabian for their technical assistance.

\section{References}

1 Tripodi A. Liver disease and hemostatic (dys)function. Semin Thromb Hemost 2015;41(05):462-467

2 Lisman T, Porte RJ. Rebalanced hemostasis in patients with liver disease: evidence and clinical consequences. Blood 2010;116 (06):878-885

3 Lisman T, Bongers TN, Adelmeijer J, et al. Elevated levels of von Willebrand Factor in cirrhosis support platelet adhesion despite reduced functional capacity. Hepatology 2006;44(01):53-61

4 Wannhoff A, Müller OJ, Friedrich K, et al. Effects of increased von Willebrand factor levels on primary hemostasis in thrombocytopenic patients with liver cirrhosis. PLoS One 2014;9(11):e112583

5 La Mura V, Reverter JC, Flores-Arroyo A, et al. Von Willebrand factor levels predict clinical outcome in patients with cirrhosis and portal hypertension. Gut 2011;60(08):1133-1138

6 Ferlitsch M, Reiberger T, Hoke M, et al. von Willebrand factor as new noninvasive predictor of portal hypertension, decompensation and mortality in patients with liver cirrhosis. Hepatology 2012;56(04):1439-1447

7 Fujikawa K, Suzuki H, McMullen B, Chung D. Purification of human von Willebrand factor-cleaving protease and its identification as a new member of the metalloproteinase family. Blood 2001;98(06):1662-1666

8 Sadler JE. Von Willebrand factor, ADAMTS13, and thrombotic thrombocytopenic purpura. Blood 2008;112(01):11-18

9 Zhou W, Inada M, Lee TP, et al. ADAMTS13 is expressed in hepatic stellate cells. Lab Invest 2005;85(06):780-788

10 Uemura M, Tatsumi K, Matsumoto $M$, et al. Localization of ADAMTS13 to the stellate cells of human liver. Blood 2005;106 (03):922-924

11 Atzori L, Poli G, Perra A. Hepatic stellate cell: a star cell in the liver. Int J Biochem Cell Biol 2009;41(8-9):1639-1642

12 Mannucci PM, Canciani MT, Forza I, Lussana F, Lattuada A, Rossi E. Changes in health and disease of the metalloprotease that cleaves von Willebrand factor. Blood 2001;98(09):2730-2735

13 Feys HB, Canciani MT, Peyvandi F, Deckmyn H, Vanhoorelbeke K, Mannucci PM. ADAMTS13 activity to antigen ratio in physiological and pathological conditions associated with an increased risk of thrombosis. Br J Haematol 2007;138(04):534-540

14 Anstee QM, Dhar A, Thursz MR. The role of hypercoagulability in liver fibrogenesis. Clin Res Hepatol Gastroenterol 2011;35(8-9):526-533

15 Papp M, Vitalis Z, Altorjay I, et al. Acute phase proteins in the diagnosis and prediction of cirrhosis associated bacterial infections. Liver Int 2012;32(04):603-611

16 Kalambokis GN, Oikonomou A, Christou L, et al. von Willebrand factor and procoagulant imbalance predict outcome in patients with cirrhosis and thrombocytopenia. J Hepatol 2016;65(05):921-928

17 Di Martino V, Weil D, Cervoni JP, Thevenot T. New prognostic markers in liver cirrhosis. World J Hepatol 2015;7(09):1244-1250

18 Raparelli V, Basili S, Carnevale R, et al. Low-grade endotoxemia and platelet activation in cirrhosis. Hepatology 2017;65(02): 571-581

19 Moreau R, Jalan R, Gines P, et al; CANONIC Study Investigators of the EASL-CLIF Consortium. Acute-on-chronic liver failure is a distinct syndrome that develops in patients with acute decompensation of cirrhosis. Gastroenterology 2013;144(07):1426-1437

20 Papp M, Tornai T, Vitalis Z, et al. Presepsin teardown - pitfalls of biomarkers in the diagnosis and prognosis of bacterial infection in cirrhosis. World J Gastroenterol 2016;22(41):9172-9185

21 Tornai T, Vitalis Z, Sipeki N, et al. Macrophage activation marker, soluble CD163, is an independent predictor of short-term mortality in patients with cirrhosis and bacterial infection. Liver Int 2016;36(11):1628-1638

22 Foldi I, Tornai T, Tornai D, et al. Lectin-complement pathway molecules are decreased in patients with cirrhosis and constitute the risk of bacterial infections. Liver Int 2017;37(07): 1023-1031 
23 Ikeda H, Tateishi R, Enooku K, et al. Prediction of hepatocellular carcinoma development by plasma ADAMTS13 in chronic hepatitis B and C. Cancer Epidemiol Biomarkers Prev 2011;20(10):2204-2211

24 Liu Y, Wang X, Li S, et al. The role of von Willebrand factor as a biomarker of tumor development in hepatitis B virus-associated human hepatocellular carcinoma: a quantitative proteomic based study. J Proteomics 2014;106:99-112

25 Cejka J. Enzyme immunoassay for factor VIII-related antigen. Clin Chem 1982;28(06):1356-1358

26 Mendelboum Raviv S, Szekeres-Csiki K, Jenei A, et al. Coating conditions matter to collagen matrix formation regarding von Willebrand factor and platelet binding. Thromb Res 2012;129 (04):e29-e35

27 Kokame K, Nobe Y, Kokubo Y, Okayama A, Miyata T. FRETSVWF73, a first fluorogenic substrate for ADAMTS13 assay. Br J Haematol 2005;129(01):93-100

28 Udvardy ML, Szekeres-Csiki K, Hársfalvi J. Novel evaluation method for densitometric curves of von Willebrand factor multimers and a new parameter $(\mathrm{M}(\mathrm{MW}))$ to describe the degree of multimersation. Thromb Haemost 2009;102(02):412-417

29 Stockschlaeder M, Schneppenheim R, Budde U. Update on von Willebrand factor multimers: focus on high-molecular-weight multimers and their role in hemostasis. Blood Coagul Fibrinolysis 2014;25(03):206-216

30 Pereboom ITA, Adelmeijer J, van Leeuwen Y, Hendriks HG, Porte RJ, Lisman T. No evidence for systemic platelet activation during or after orthotopic liver transplantation. Liver Transpl 2009;15 (08):956-962

31 Shenkman B, Savion N, Dardik R, Tamarin I, Varon D. Testing of platelet deposition on polystyrene surface under flow conditions by the cone and plate(let) analyzer: role of platelet activation, fibrinogen and von Willebrand factor. Thromb Res 2000;99(04): 353-361

32 Vanícková M, Suttnar J, Dyr JE. The adhesion of blood platelets on fibrinogen surface: comparison of two biochemical microplate assays. Platelets 2006;17(07):470-476

33 Tornai I, Hársfalvi J, Boda Z, Udvardy M, Pfliegler G, Rak K. Endothelium releases more von Willebrand factor and tissue-type plasminogen activator upon venous occlusion in patients with liver cirrhosis than in normals. Haemostasis 1993;23(01):58-64

34 Ferro D, Quintarelli C, Lattuada A, et al. High plasma levels of von Willebrand factor as a marker of endothelial perturbation in cirrhosis: relationship to endotoxemia. Hepatology 1996;23(06):1377-1383

35 Uemura M, Fujimura Y, Matsumoto $M$, et al. Comprehensive analysis of ADAMTS13 in patients with liver cirrhosis. Thromb Haemost 2008;99(06):1019-1029
36 Hugenholtz GC, Adelmeijer J, Meijers JC, Porte RJ, Stravitz RT, Lisman T. An unbalance between von Willebrand factor and ADAMTS13 in acute liver failure: implications for hemostasis and clinical outcome. Hepatology 2013;58(02):752-761

37 Pereboom IT, Adelmeijer J, van Leeuwen Y, Hendriks HG, Porte RJ, Lisman T. Development of a severe von Willebrand factor/ ADAMTS13 dysbalance during orthotopic liver transplantation. Am J Transplant 2009;9(05):1189-1196

38 Favaloro EJ, Bonar R, Chapman K, Meiring M, Funk Adcock D. Differential sensitivity of von Willebrand factor (VWF) 'activity' assays to large and small VWF molecular weight forms: a crosslaboratory study comparing ristocetin cofactor, collagen-binding and mAb-based assays. J Thromb Haemost 2012;10(06): 1043-1054

39 Fisher C, Patel VC, Stoy SH, et al. Balanced haemostasis with both hypo- and hyper-coagulable features in critically ill patients with acute-on-chronic-liver failure. J Crit Care 2018;43:54-60

40 Federici AB, Berkowitz SD, Lattuada A, Mannucci PM. Degradation of von Willebrand factor in patients with acquired clinical conditions in which there is heightened proteolysis. Blood 1993;81 (03):720-725

41 Lancellotti S, De Filippis V, Pozzi N, et al. Oxidized von Willebrand factor is efficiently cleaved by serine proteases from primary granules of leukocytes: divergence from ADAMTS-13. J Thromb Haemost 2011;9(08):1620-1627

42 Carnevale R, Raparelli V, Nocella C, et al. Gut-derived endotoxin stimulates factor VIII secretion from endothelial cells. Implications for hypercoagulability in cirrhosis. J Hepatol 2017;67(05): 950-956

43 Villa E, Cammà C, Marietta M, et al. Enoxaparin prevents portal vein thrombosis and liver decompensation in patients with advanced cirrhosis. Gastroenterology 2012;143(05):1253-1260

44 Reuken PA, Kussmann A, Kiehntopf M, et al. Imbalance of von Willebrand factor and its cleaving protease ADAMTS13 during systemic inflammation superimposed on advanced cirrhosis. Liver Int 2015;35(01):37-45

45 Kleinegris MC, Bos MH, Roest M, et al. Cirrhosis patients have a coagulopathy that is associated with decreased clot formation capacity. J Thromb Haemost 2014;12(10):1647-1657

46 Cao WJ, Niiya M, Zheng XW, Shang DZ, Zheng XL. Inflammatory cytokines inhibit ADAMTS13 synthesis in hepatic stellate cells and endothelial cells. J Thromb Haemost 2008;6(07):1233-1235

47 Furlan M, Robles R, Morselli B, Sandoz P, Lämmle B. Recovery and half-life of von Willebrand factor-cleaving protease after plasma therapy in patients with thrombotic thrombocytopenic purpura. Thromb Haemost 1999;81(01):8-13 